\title{
OBTENÇÃO EM LARGA ESCALA DE TRANSMISSORES DE PRESSÃO PIEZORESISTIVOS DE ALTO DESEMPENHO
}

\author{
CESAR A. MAYOR ${ }^{1}$, EDGAR CHARRY ${ }^{1}$.
}

1. Laboratório de Sistemas Integráveis, Escola Politécnica, Universidade de São Paulo Av. Prof. Luciano Gualberto Trav.3 N.158. CEP:05508-900, São Paulo, SP, BRASIL E-mails: cesar.augusto.mayor@usp.br, charry@lsi.usp.br

\begin{abstract}
Is presented a system for time optimization of the calibration and compensation process of piezoresistive pressure sensors for automation and control applications with high performance characteristics, with compensation of several error sources, specially, compensation of temperature dependencies and nonlinearity.
\end{abstract}

Keywords_- Sensors for automation, instrumentation, sensor calibration, error compensation.

\begin{abstract}
Resumo-É apresentado um sistema para otimização do tempo de calibração e compensação de sensores piezoresistivos de pressão para aplicações de automação e controle com características de alto desempenho, com compensação de diversas fontes de erro, especialmente, compensação de temperatura e não-linearidade.
\end{abstract}

Palavras-chave— Sensores para Automação, instrumentação, calibração de sensores, compensação do erro.

\section{Introdução}

Os Transmissores de Pressão são elementos de medição que pertencem à área de automação industrial, que por sua vez faz parte de um segmento da economia do país conhecida como indústria eletroeletrônica. Os sensores objeto de estudo desta pesquisa são sensores de pressão piezoresistivos com condicionamento de sinal para obter uma saída em corrente de dois fios de 4-20mA. Nos dispositivos estudados, o processo de calibração e compensação do sinal do sensor de pressão é realizado de forma completamente digital, utilizando o MAX1464 (Maxim, 02-2005) que é um chip ASIC (Application Specific Integrated Circuit) de condicionamento de sinal.

A produção de um transdutor de pressão baseado em um sensor piezoresistivo tem que levar em conta vários fatores relacionados com a exatidão para poder fazer com que a medição da variável do processo e sua correspondente representação elétrica estejam próximo ao valor real. Na Figura 1 são ilustrados os fatores relacionados com a exatidão do sistema. Essas fontes de erro são inerentes ao transdutor, mas a magnitude do erro de cada uma delas pode depender das entradas principais e menores do sistema.

O objetivo é produzir um transmissor em conformidade com as normas internacionais para transmissores para uso em sistemas de controle de processos industriais IEC 60770 (CENELEC, 1999), (CENELEC, 1995).

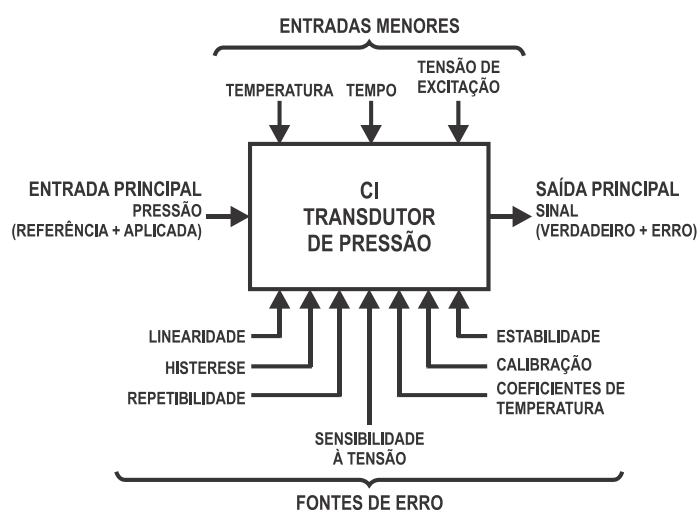

Figura 1. Parâmetros de desempenho do transdutor relacionados com a exatidão.

A norma especifica métodos de teste uniformes para a avaliação do desempenho de transmissores com sinais de saída elétricos ou pneumáticos, definindo os fatores relacionados com a exatidão (estabilidade, calibração, histerese, repetibilidade, linearidade, dependência de temperatura, etc) para poder obter as características de erro total. As superfícies ilustradas nas figuras 2 e 3 também são conhecidas como "Total Error Band (TEB)", ou seja, a menor faixa de erro que cobre todos os valores de erro em função da pressão e da temperatura dentro da faixa de operação. O comportamento da saída de um sensor piezoresistivo sem compensar e calibrar é ilustrado na Figura 2. O objetivo do processo de calibração e compensação é diminuir o erro de saída e obter uma superfície similar à mostrada na Figura 3. 


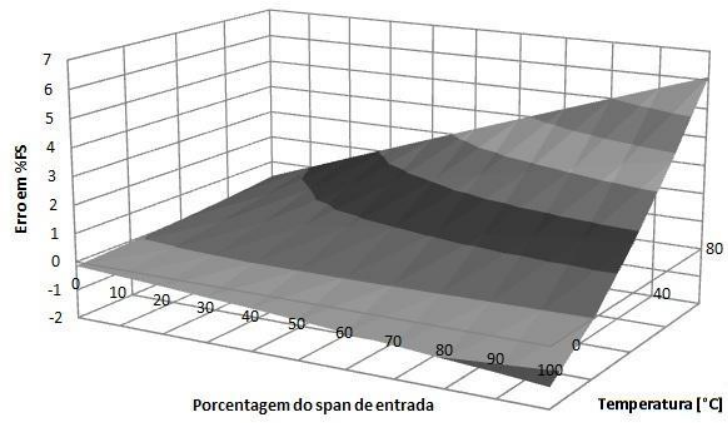

Figura 2. Sensor sem calibrar e compensar: saída vs entrada em $\%$ FS e vs temperatura.

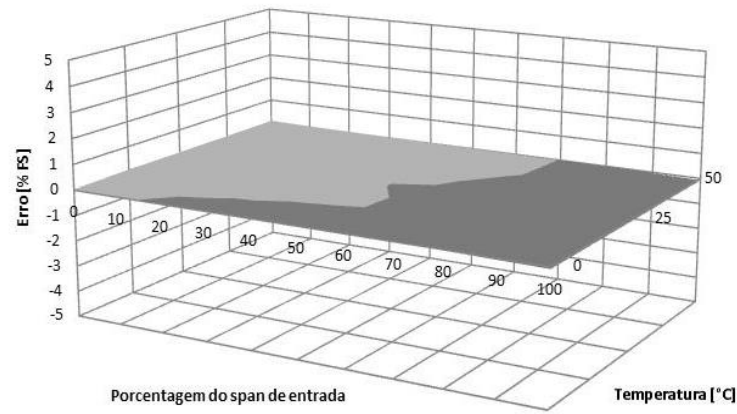

Figura 3. Sensor calibrado e compensado: erro na saída vs entrada em $\%$ FS e vs temperatura.

\subsection{Processo de Calibração e Compensação}

O processo de correção das características do sensor está dividido em duas etapas fundamentais: Uma delas é executada durante a fabricação do transmissor e consiste em obter numa bancada de calibração os coeficientes específicos que corrigem as características do sensor e do MAX1464. Estes coeficientes são específicos para cada conjunto sensor-MAX1464. O segundo momento é executado quando o transmissor está medindo, e consiste em executar em tempo real as funções de correção dos dados medidos pelo sensor e fornecer a saída ou resultado da medição corrigida usando os coeficientes que foram calculados na fase de calibração e compensação (Maxim, 09-2005).

Os transmissores passam por uma fase de aquisição de dados a três temperaturas e três pressões, tomando dados do $\mathrm{AD}$ do sensor de pressão, do $\mathrm{AD}$ do sensor de temperatura e também tomando dados para dois pontos de corrente da saída correspondentes a dois valores de configuração do DAC, para gerar uma matriz como a ilustrada na Tabela 1.

Tabela 1.Matriz de medições do processo de calibração e compensação.

\begin{tabular}{|l|l|l|l|l|l|l|}
\hline & Pmin & Pmed & Pmax & Temp & Iout $_{1}$ & Iout $_{2}$ \\
\hline Tmin & $\operatorname{Adc}_{1,1}$ & $\operatorname{Adc}_{1,2}$ & $\operatorname{Adc}_{1,3}$ & Tadc $_{1}$ & Iout $_{1,1}$ & Iout $_{1,2}$ \\
\hline Tmed & $\operatorname{Adc}_{2,1}$ & $\operatorname{Adc}_{2,2}$ & $\operatorname{Adc}_{2,3}$ & Tadc $_{2}$ & Iout $_{2,1}$ & Iout $_{2,2}$ \\
\hline Tmax & $\operatorname{Adc}_{3,1}$ & $\operatorname{Adc}_{3,2}$ & $\operatorname{Adc}_{3,3}$ & Tadc $_{3}$ & Iout $_{3,1}$ & Iout $_{3,2}$ \\
\hline
\end{tabular}

As temperaturas e pressões às que são submetidos os transmissores no processo de medição são as que determinam a faixa de operação. Por exemplo, se o objetivo do transmissor é fornecer uma saída calibrada e compensada de Obar a 10bar para funcionar em um ambiente com temperaturas entre $0^{\circ} \mathrm{C}$ e $50^{\circ} \mathrm{C}$, os valores para Tmin, Tmed, Tmax, Pmin, Pmed e Pmax seriam:

$$
\begin{aligned}
& \text { Tmin }=0{ }^{\circ} \mathrm{C}, \text { Tmed }=25^{\circ} \mathrm{C}, \text { Tmax }=50^{\circ} \mathrm{C} \\
& \text { Pmin }=0 \text { bar, Pmed }=5 \text { bar, } P \max =10 \text { bar }
\end{aligned}
$$

Para adquirir os dados é usada uma interface de usuário em Labview. A interface permite configurar e ler os registradores do MAX1464, tendo acesso às leituras do $\mathrm{AD}$ e conseguindo também configurar o DAC para obter qualquer saída desejada dentro dos limites permitidos.

O sistema de aquisição de dados fornecido pela MAXIM (fabricantes do MAX1464) permite realizar o processo de calibração e compensação para um único transmissor de pressão por vez, e esse processo demora aproximadamente duas horas e meia para cada temperatura, somando um total de aproximadamente 7 a 8 horas para o processo inteiro. Isto devido a que para poder obter medições confiáveis é necessário esperar que todas as partes do transmissor (sensor, dispositivos eletrônicos, conector de processo e peças de proteção e isolamento do meio) tenham atingido a estabilização térmica antes de efetuar as medições.

\section{Proposta de sistema de Multiplexação}

Devido à quantidade de tempo que leva para compensar e calibrar cada transmissor de pressão foi necessário projetar um sistema de aquisição de dados que permitisse maximizar o tempo de estabilização térmica e efetuar medições de vários transmissores de pressão simultaneamente.

O trabalho desenvolvido nesta etapa é de vital importância para o melhoramento do sistema geral e para incrementar o número de transmissores que podem ser calibrados. Consiste basicamente no projeto de um sistema de multiplexação assim como o protocolo de comunicações para fazer o controle desde uma interface de usuário em Labview.

\subsection{Características do sistema de Multiplexação}

O hardware projetado efetua a multiplexação dos sinais necessários para o processo de compensação e calibração dos transmissores de pressão objetos do estudo. Os sinais multiplexados são os seguintes: comunicação SPI (5 fios: VDD, DGND, CS, DIO, SCLK) e alimentação do transmissor (2 fios: positivo e negativo da fonte de tensão). É importante lembrar que o negativo da fonte de tensão e o GND da comunicação SPI são sinais diferentes devido às características do hardware do transmissor de pressão (Fernandez da Luz, 2006).

O diagrama de blocos dos sinais, a fonte de alimentação e o PC esta ilustrado na figura 4. 


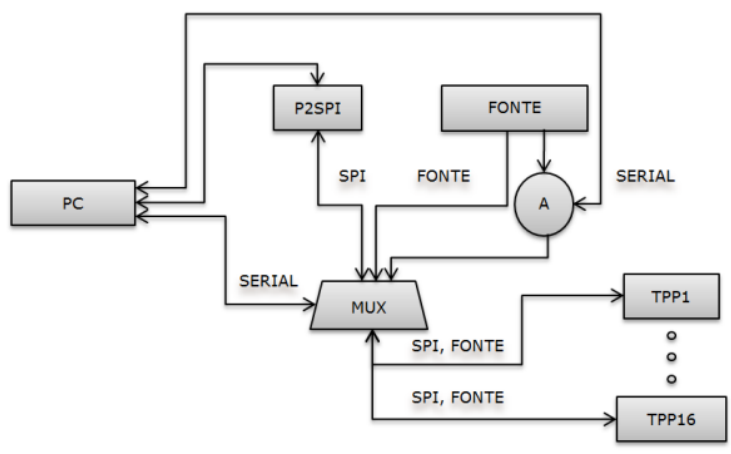

Figura 4. Diagrama de Blocos do sistema de multiplexação

Na figura 4: (A) representa um multímetro em modo de medição de corrente, (MUX) representa o multiplexor projetado, (TPPx) representa cada transmissor de pressão e (P2SPI) representa o módulo da MAXIM para fazer a conversão de paralelo a SPI.

Os sinais multiplexados são os correspondentes ao protocolo SPI (5 fios) e à alimentação do transmissor (2 fios). Para o SPI todas a tensões estão na faixa $0-5 \mathrm{~V}$. O consumo de corrente no transmissor não é influenciado pela conexão SPI já que quando são realizadas as medições a porta SPI não é usada; ela é usada só para configuração dos registradores ou para programar a memória Flash do MAX1464. Para a alimentação do transmissor as tensões estão entre 12-32 V. A corrente mínima é de $4 \mathrm{~mA}$ e a máxima é de $20 \mathrm{~mA}$.

\subsection{Protocolo Serial no Microcontrolador do Sistema de Multiplexação}

O protocolo de comunicações entre o sistema de Multiplexação e a interface de usuário em Labview usa RS232. O diagrama de fluxo do software programado no microcontrolador do sistema de multiplexação é ilustrado na figura 5 .

O sistema proposto permite uma comunicação suficientemente rápida e confiável para enviar os dados de configuração, comandos e mensagens de estado e erro entre o sistema Multiplexador e a interface de usuário.

\subsection{Interface de usuário no Labview.}

A componente mais forte no trabalho desenvolvido foi a elaboração do software em Labview que serve como interface de usuário, para fazer a compensação e calibração de até 16 transmissores de pressão. A interface de usuário foi elaborada para dar acesso ao usuário a todas as funções de configuração, medição, geração de coeficientes e programação do MAX1464, usando como base a interface de usuário para um único transmissor fornecida pela MAXIM. A figura 6 ilustra o painel frontal da interface de usuário modificada para o sistema com multiplexação.

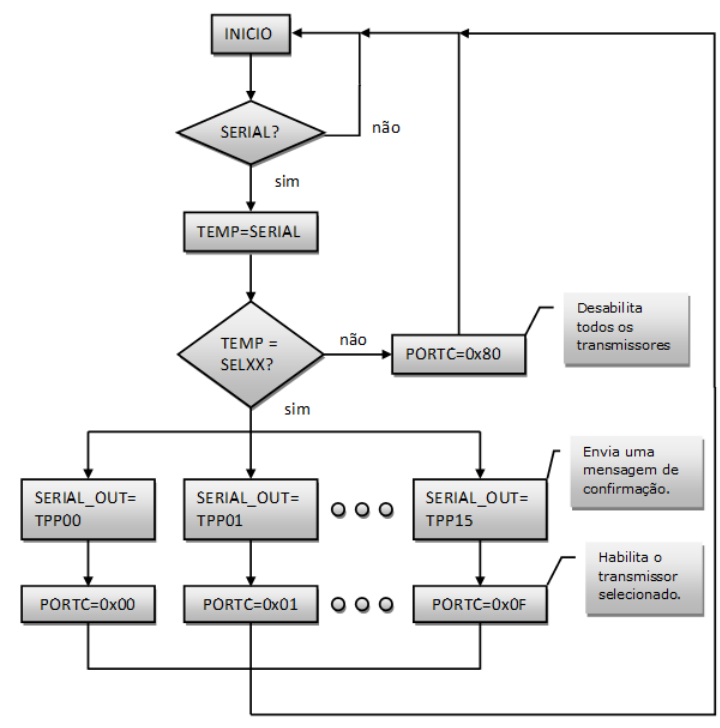

Figura 5. Diagrama de fluxo do software programado no microcontrolador do sistema de multiplexação.

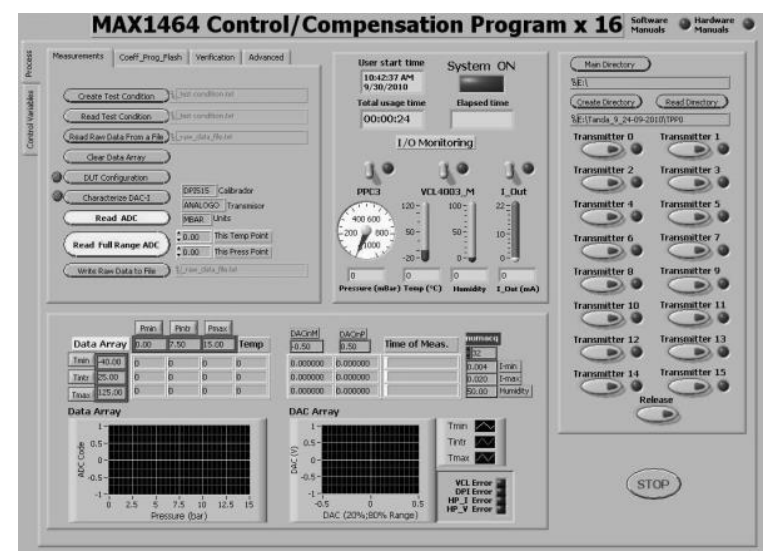

Figura 6. Painel frontal da interface de usuário.

A interface de usuário está dividida em quatro módulos que contêm todas as opções necessárias para fazer o processo de calibração e compensação. $\mathrm{O}$ primeiro módulo contém as opções para efetuar as medições, a geração de coeficientes, programação, configuração e verificação. O segundo módulo contém informações do processo e indicadores do calibrador de pressão, temperatura e umidade da câmara climática e corrente de saída do transmissor de pressão selecionado. O terceiro módulo contém os controles para criar ou ler pastas de trabalho e para ativar ou desativar qualquer uma das dezesseis saídas do multiplexador. E o quarto módulo contém as matrizes e gráficos dos dados medidos.

\section{Procedimento de calibração e compensação usando a interface de usuário}

Existem três fases no procedimento de calibração e compensação. A primeira fase é de configuração do sistema, a segunda é de toma de dados e a terceira é de geração de coeficientes e programação do transmissor. 


\subsection{Configuração do Sistema}

$\mathrm{Na}$ fase de configuração do sistema o usuário tem que criar as pastas de trabalho de cada transmissor onde serão armazenados os arquivos de condições de teste, medições, coeficientes gerados e arquivos HEX para programar o MAX1464.

Posteriormente, devem ser configurados os registradores de cada MAX1464 de cada transmissor de pressão com os valores padrão. O valor do registrador que configura o ganho do PGA (Amplificador de Ganho Programável) deve ser ajustado para obter um melhor desempenho e maximizar a faixa de operação do conversor AD do MAX1464. Para isso deve ser selecionada a temperatura para a qual a sensibilidade do sensor é maior, e aplicar pressão máxima e mínima em um processo iterativo até obter o ganho que maximize a saída do AD (Charry, 2007). Na Figura 7 é ilustrado o diagrama de fluxo desta fase.

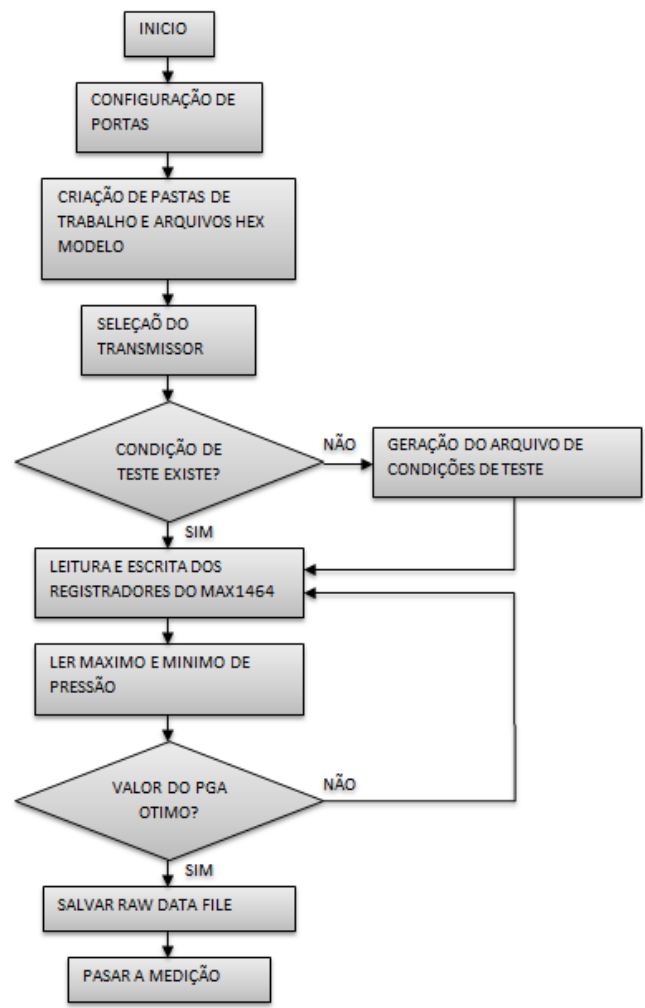

Figura 7. Diagrama de fluxo da configuração do sistema

\subsection{Procedimento de Medição}

$\mathrm{Na}$ fase de medições o usuário tem que ajustar os pontos de temperatura na câmara climática e esperar a estabilização térmica para posteriormente fazer medições das diferentes pressões e temperatura do MAX1464. Esta fase demora aproximadamente 2 horas e meia para cada uma das três temperaturas, dependendo da quantidade de transmissores de pressão medidos. Na Figura 8 é ilustrado o diagrama de fluxo desta fase.

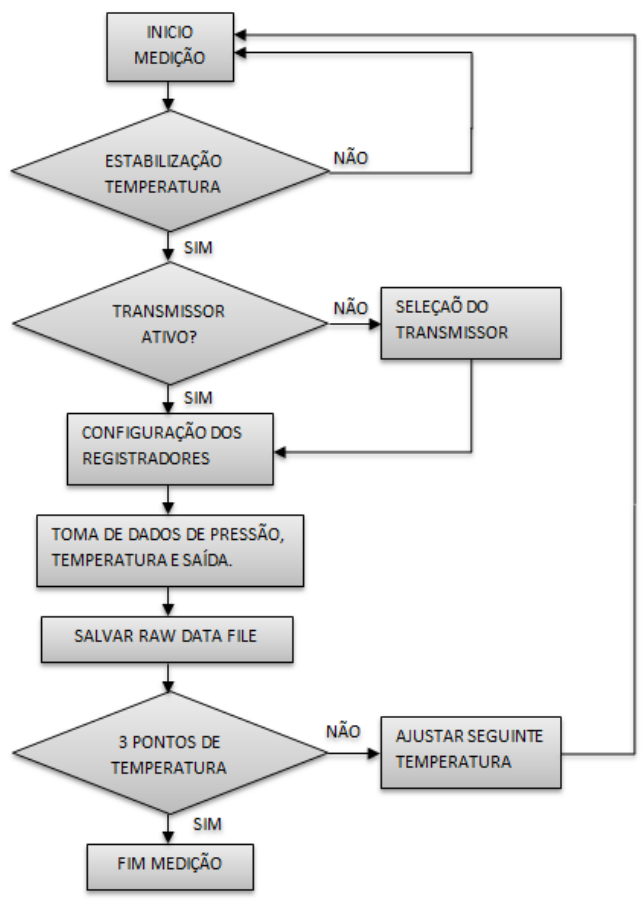

Figura 8. Diagrama de fluxo do procedimento de medição

\subsection{Geração de coeficientes e programação do transmissor de pressão}

Esta fase exige que o arquivo de medições "Raw data file" já tenha sido gerado previamente, já que ele contém as medições de pressão e temperatura e os valores dos registradores com que foram feitas essas medições. Na Figura 9 é ilustrado o diagrama de fluxo desta fase.

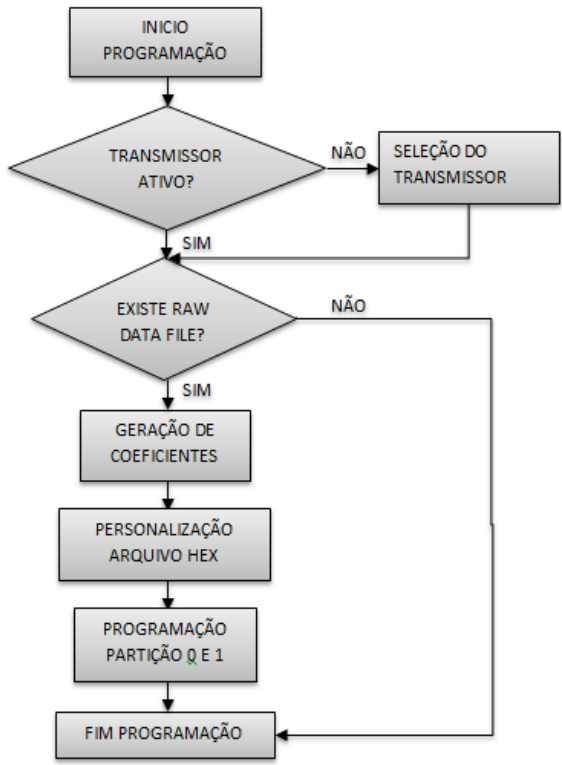

Figura 9. Diagrama de fluxo da geração dos coeficientes e programação do MAX1464

Os dados contidos no arquivo "Raw data file" são usados para gerar os coeficientes de compensação e calibração para cada transmissor de pressão. Esses coeficientes são incluídos no arquivo "HEX" de pro- 
gramação de cada MAX1464 para ser posteriormente programados, deixando eles prontos para testes e funcionamento normal.

\section{Resultados}

Os testes para avaliar o sistema foram feitos com sensores de pressão piezoresistivos antes e depois de serem integrados com os circuitos de condicionamento de sinal que conformam os transmissores de pressão, e antes de passar pelo procedimento de compensação e calibração, para poder comparar os resultados do procedimento e do sistema desenvolvido.

Os sensores usados para os testes foram submetidos ao procedimento de medição descrito na norma IEC 60770, usando o software de aquisição de dados programado em LabView e submetendo os sensores a condições controladas de temperatura e humidade. Foram usados 4 sensores Keller PR 9FL, com faixa de pressão de 20bar, submetidos a excitação constante de $1 \mathrm{~mA}$. As características dos 4 sensores antes da calibração e compensação obtidas seguindo o processo de medição estão resumidas na tabela 2.

Tabela 2. Características dos sensores medidos antes de calibrar e compensar.

\begin{tabular}{|l|c|c|c|c|}
\hline & $\begin{array}{c}\text { Sensor } \\
1\end{array}$ & $\begin{array}{c}\text { Sensor } \\
2\end{array}$ & $\begin{array}{c}\text { Sensor } \\
3\end{array}$ & $\begin{array}{c}\text { Sensor } \\
4\end{array}$ \\
\hline $\begin{array}{l}\text { Não Linearidade } \\
\text { [\%FS saída] }\end{array}$ & 0.042 & 0.035 & 0.043 & 0.043 \\
\hline $\begin{array}{l}\text { Histerese [\%FS } \\
\text { saída] }\end{array}$ & 0.011 & 0.041 & 0.030 & 0.012 \\
\hline $\begin{array}{l}\text { Repetividade } \\
{[\% \text { FS saída] }}\end{array}$ & 0.080 & 0.074 & 0.067 & 0.091 \\
\hline $\begin{array}{l}\text { TCS [\%FS saí- } \\
\text { da/(\%FS entra- } \\
\text { da* }{ }^{\circ} \text { C)] }\end{array}$ & 0.094 & 0.089 & 0.089 & 0.096 \\
\hline $\begin{array}{l}\text { TCO [\%FS saí- } \\
\text { da/ }{ }^{\circ} \text { C] }\end{array}$ & 0.774 & 0.997 & 2.222 & 0.198 \\
\hline $\begin{array}{l}\text { Erro médio [\%FS } \\
\text { saída] }\end{array}$ & 1.118 & 0.697 & 0.666 & 1.018 \\
\hline $\begin{array}{l}\text { Total Error Band } \\
{[\% \text { FS saída] }}\end{array}$ & 6.150 & 4.746 & 4.752 & 5.917 \\
\hline
\end{tabular}

A figura 10 ilustra o resultado das medições dos sensores de pressão antes do processo de calibração e compensação.

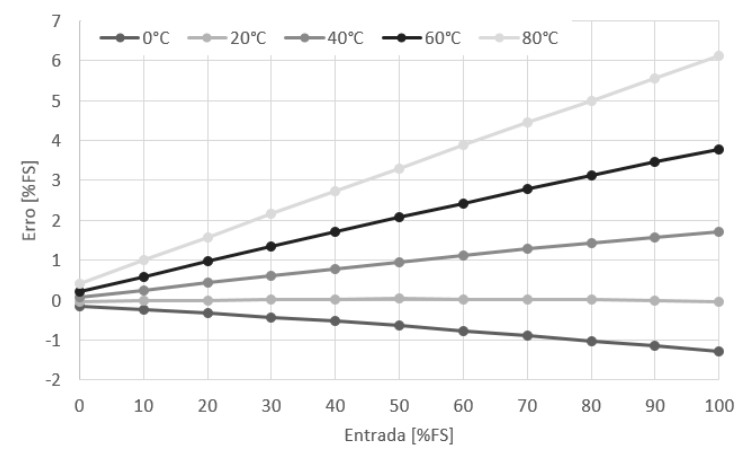

Figura 10. Saída do sensor comparada com saída à temperatura de referencia $\left(20^{\circ} \mathrm{C}\right)$ vs entrada em porcentagem de fundo de escala.

As características dos 4 transmissores de pressão depois de passar pelo processo de calibração e com- pensação obtidas seguindo o processo de medição estão resumidas na tabela 3 .

Tabela 3. Características dos transmissores de pressão medidos depois do de calibrar e compensar usando o sistema de multiplexação.

\begin{tabular}{|l|c|c|c|c|}
\hline & $\begin{array}{c}\text { Sensor } \\
1\end{array}$ & $\begin{array}{c}\text { Sensor } \\
2\end{array}$ & $\begin{array}{c}\text { Sensor } \\
3\end{array}$ & $\begin{array}{c}\text { Sensor } \\
4\end{array}$ \\
\hline $\begin{array}{l}\text { Não Linearidade } \\
\text { [\%FS saída] }\end{array}$ & 0.022 & 0.024 & 0.020 & 0.026 \\
\hline $\begin{array}{l}\text { Histerese [\%FS } \\
\text { saída] }\end{array}$ & 0.028 & 0.016 & 0.012 & 0.011 \\
\hline $\begin{array}{l}\text { Repetividade } \\
{[\% \text { FS saída] }}\end{array}$ & 0.023 & 0.024 & 0.012 & 0.014 \\
\hline $\begin{array}{l}\text { TCS [\%FS saí- } \\
\text { da/(\%FS entra- } \\
\text { da*º }{ }^{\circ} \text { ] }\end{array}$ & 0.001 & 0.001 & 0.001 & 0.001 \\
\hline $\begin{array}{l}\text { TCO [\%FS saí- } \\
\text { da/ }{ }^{\circ} \text { C] }\end{array}$ & 0.091 & 0.089 & 0.136 & 0.025 \\
\hline $\begin{array}{l}\text { Erro médio [\%FS } \\
\text { saída] }\end{array}$ & 0.059 & 0.076 & 0.081 & 0.062 \\
\hline $\begin{array}{l}\text { Total Error Band } \\
{[\% F S \text { saída] }}\end{array}$ & 0.06 & 0.087 & 0.098 & 0.082 \\
\hline
\end{tabular}

A figura 11 ilustra o resultado das medições dos transmissores de pressão compensados e calibrados usando o sistema de multiplexação.

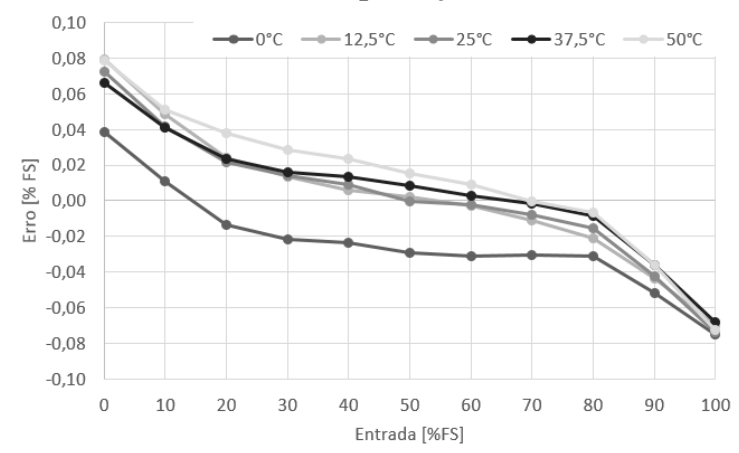

Figura 11. Erro da saída dos transmissores de pressão comparada com a saída ideal depois de ser calibrados e compensados com o sistema de multiplexação.

O tempo total do processo de calibração e compensação foi registrado comparando o sistema com e sem multiplexação. Os resultados são ilustrados na figura 12.

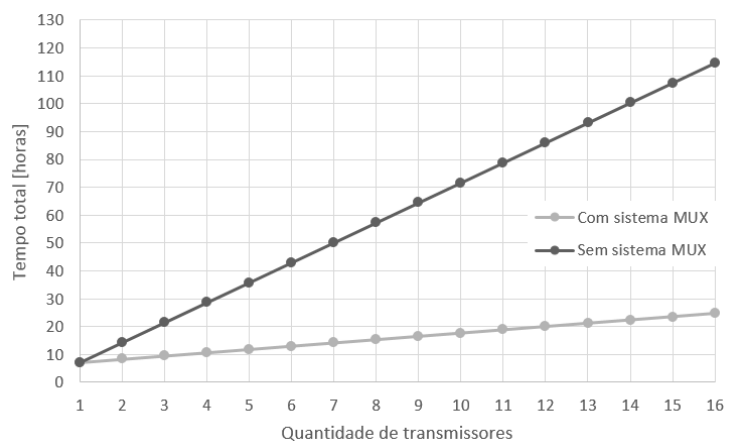

Figura 12. Tempo total do processo de compensação e calibração com e sem o sistema de multiplexação vs quantidade de transmissores. 


\section{Discussões}

Como pode ser observado comparando os resultados das medições das tabelas 2 e 3, e das figuras 10 e 11 , os sensores antes de ser compensados e calibrados apresentam uma alta dependência com a temperatura, além de apresentar características como não linearidade e histerese. No caso da não linearidade e da histerese é observado na média uma diminuição dessas grandezas, mas o caso mais notável é a diminuição da dependência da sensibilidade e do offset em relação à temperatura. Para os sensores sem compensar e calibrar o erro médio é de quase $1 \%$ FS e o TEB chega até $6 \%$ FS. Já para as medições dos transmissores compensados e calibrados o erro médio é aproximadamente $0,07 \% \mathrm{FS}$, enquanto o TEB não supera $\mathrm{o}$ $0,1 \% \mathrm{FS}$.

Esses resultados coincidem com a diminuição do TCO (coeficiente de temperatura do zero) e TCS (coeficiente de temperatura da sensibilidade) devido ao processo de calibração e compensação. Para os sensores sem calibrar e compensar o TCO chega a ter mais de $2 \%\left[\mathrm{FSsaída} /{ }^{\circ} \mathrm{C}\right]$ e o $\mathrm{TCS}$ chega até $0,1 \%\left[\right.$ FSsaída $/\left(\%\right.$ FSentrada $\left.\left.{ }^{* \circ} \mathrm{C}\right)\right]$. Já para os transmissores compensados e calibrados o TCO é aproximadamente $0,1 \%\left[\mathrm{FSsaída} /{ }^{\circ} \mathrm{C}\right]$ e o TCS de aproximadamente $0,001 \%\left[\right.$ FSsaída/(\%FSentrada $\left.\left.{ }^{* \circ} \mathrm{C}\right)\right]$, significando uma grande diminuição da dependência dos transmissores à temperatura.

Os resultados das características dos transmissores de pressão calibrados e compensados usando o sistema de multiplexação são iguais aos obtidos com o sistema original fornecido pela MAXIM (calibração e compensação de um único transmissor por vez), demonstrando que o sistema projetado não interfere nas medições e permite que os transmissores de pressão cumpram com as características de desempenho desejadas, ou seja, manter a saída com TEB menor que $0,1 \% \mathrm{FS}$.

\subsection{Problemas associados às medições}

No procedimento de medição podem se apresentar vários problemas devidos a fatores externos e de manipulação do transmissor que influenciam na confiabilidade dos dados medidos. Estes problemas estão relacionados principalmente com o tempo necessário de estabilização térmica antes de efetuar a medição. As diferenças térmicas influenciam principalmente as medições de saída de corrente para mudanças nos valores do DAC e as medições do $\mathrm{ADC}$ do sensor de temperatura.

\section{Conclusão}

Os transmissores calibrados e compensados usando o sistema de multiplexação projetado cumprem com as características de desempenho desejadas, iguais ou melhores que as atingidas pelo sistema de compensa- ção e calibração simples (de um único transmissor de pressão por vez).

O tempo total do processo de compensação e calibração de mais de um transmissor foi reduzido em aproximadamente 6 horas por cada transmissor adicional até um máximo de 16 transmissores simultâneos. No caso de 16 transmissores o sistema sem multiplexação demoraria 114 horas contra 24 horas do sistema com multiplexação.

O sistema de multiplexação projetado permite produzir transmissores de pressão com características de alto desempenho com uma redução muito significativa no tempo total do processo.

Com futuras versões do sistema de multiplexação seria interessante efetuar uma verificação automática da estabilidade das medições em função do tempo de estabilização térmica para evitar possíveis erros e aumentar a confiabilidade de cada medição. Adicionalmente seria interessante diminuir ainda mais a intervenção do usuário para deixar o sistema mais automatizado.

\section{Agradecimentos}

Os autores agradecem ao suporte financeiro do Conselho Nacional de Desenvolvimento Cientifico e Tecnológico - CNPq, ao Laboratório de Sistemas Integráveis - LSI da Universidade de São Paulo, à MEMS - Microssistemas Integrados Híbridos de Pressão Indústria e Comércio Ltda e ao professor Edgar Charry pelas discussões e instruções referentes à parte técnica deste trabalho.

\section{Referências Bibliográficas}

CENELEC, 1995. IEC 61298 - 1 Process measurement and control devices - General methods and procedures for evaluating performance: General considerations.

CENELEC, 1999. BS EN 60770 - Transmitters for use in industrial process control systems: Methods for performance evaluation.

Charry, E., 2007. Verificação dos limítes térmicos do Transmissor de Pressão MEMS-641series, São Paulo.

Fernandez da Luz, S., 2006. Microssistema Transmissor de Pressão Piezorressistivo de 420mA de Alta Precisão, São Paulo.

Maxim, 02-2005. MAX1464 Low-Power, Low-Noise Multichannel Sensor Signal Processor, Overview. [Online] Disponível em: http://data sheets.maximintegrated.com/en/ds/MAX1464.pd f [Acesso em 20 Fevereiro 2013].

Maxim, 09-2005. MAX1464 Signal-Conditioner, Sensor Compensation Algorithm, Application Note 3649. [Online] Disponível em: http://pdfserv.maximintegrated.com/en/an/AN36 49.pdf [Acesso em 10 Fevereiro 2013]. 